\title{
CPTAC Bladder Urothelial Carcinoma Baseline Form
}

National Cancer Institute

\section{Source}

National Cancer Institute. CPTAC Bladder Urothelial Carcinoma Baseline Form. NCI

Thesaurus. Code C157524.

Terminology used in support of the data collection efforts of the Clinical Proteomic

Tumor Analysis Consortium (CPTAC) with the focus on Bladder Urothelial Carcinoma Baseline Form data. 